\title{
Perelman's invariant, Ricci flow, and the Yamabe invariants of smooth manifolds
}

\author{
Kazuo Akutagawa, Masashi Ishida and Claude LeBrun*
}

\begin{abstract}
In his study of Ricci flow, Perelman introduced a smooth-manifold invariant called $\bar{\lambda}$. We show here that, for completely elementary reasons, this invariant simply equals the Yamabe invariant, alias the sigma constant, whenever the latter is non-positive. On the other hand, the Perelman invariant just equals $+\infty$ whenever the Yamabe invariant is positive.
\end{abstract}

Mathematics Subject Classification (2000). Primary 53C21; Secondary 58J50.

Keywords. Scalar curvature, Ricci flow, conformal geometry, Perelman invariant, Yamabe problem.

Let $M$ be a smooth compact manifold of dimension $n \geq 3$. Perelman's celebrated work on Ricci flow $[12,13]$ led him to consider the functional which associates to every Riemannian metric $g$ the least eigenvalue $\lambda_{g}$ of the elliptic operator $4 \Delta_{g}+s_{g}$, where $s_{g}$ denotes the scalar curvature of $g$, and $\Delta=d^{*} d=-\nabla \cdot \nabla$ is the positive-spectrum Laplace-Beltrami operator associated with $g$. In other words, $\lambda_{g}$ can be expressed in terms of Raleigh quotients as

$$
\lambda_{g}=\inf _{u} \frac{\int_{M}\left[s_{g} u^{2}+4|\nabla u|^{2}\right] d \mu}{\int_{M} u^{2} d \mu}
$$

where the infimum is taken over all smooth, real-valued functions $u$ on $M$.

One of Perelman's remarkable observations is that the scale-invariant quantity $\lambda_{g} V_{g}^{2 / n}$ is non-decreasing under the Ricci flow, where $V_{g}=\int_{M} d \mu_{g}$ denotes the total volume of $(M, g)$. This led him to consider the differential-topological invariant obtained by taking the supremum of this quantity over the space of all Riemannian metrics $[13,6]$ :

\footnotetext{
*Supported in part by NSF grant DMS-0604735.
} 
Definition 1. Let $M$ be a smooth compact $n$-manifold, $n \geq 3$. Perelman's $\bar{\lambda}$ invariant of $M$ is defined to be

$$
\bar{\lambda}(M)=\sup _{g} \lambda_{g} V_{g}^{2 / n}
$$

where the supremum is taken over all smooth metrics $g$ on $M$.

The rôle of the scalar curvature and Laplacian in defining $\lambda_{g}$ might immediately make one wonder whether this invariant might somehow be related to the Yamabe problem. Recall that, as was conjectured by Yamabe [21], and later proved by Trudinger, Aubin, and Schoen $[2,3,11,18,20]$, every conformal class on any smooth compact manifold contains a metric of constant scalar curvature. If $M$ is a smooth compact manifold of dimension $n \geq 3$, and if

$$
\gamma=[g]=\left\{v g \mid v: M \rightarrow \mathbb{R}^{+}\right\},
$$

is the conformal class of an arbitrary metric, such a metric $\hat{g}$ can in fact be constructed by minimizing the normalized total-scalar-curvature functional

$$
\hat{g} \mapsto \frac{\int_{M} s_{\hat{g}} d \mu_{\hat{g}}}{\left(\int_{M} d \mu_{\hat{g}}\right)^{\frac{n-2}{n}}},
$$

among all metrics conformal to $g$. Indeed, by setting $\hat{g}=u^{4 /(n-2)} g$, this expression can be rewritten as

$$
\frac{\int_{M} s_{\hat{g}} d \mu_{\hat{g}}}{\left(\int_{M} d \mu_{\hat{g}}\right)^{\frac{n-2}{n}}}=\frac{\int_{M}\left[s_{g} u^{2}+4 \frac{n-1}{n-2}|\nabla u|^{2}\right] d \mu_{g}}{\left(\int_{M} u^{2 n /(n-2)} d \mu_{g}\right)^{(n-2) / n}},
$$

and the proof proceeds by showing that there is a smooth positive function $u$ which minimizes the right-hand expression. In particular, each conformal class $\gamma$ has an associated number $Y_{\gamma}$, called its Yamabe constant, obtained by setting

$$
Y_{\gamma}=\inf _{g \in \gamma} \frac{\int_{M} s_{g} d \mu_{g}}{\left(\int_{M} d \mu_{g}\right)^{\frac{n-2}{n}}} ;
$$

the content of the Trudinger-Aubin-Schoen theorem is exactly that this number is actually realized as the constant scalar curvature of some unit-volume metric in $\gamma$. A constant-scalar-curvature metric of this type is called a Yamabe minimizer. It is not difficult to show that any Riemannian metric with $s=$ const $\leq 0$ is a Yamabe minimizer, and that, moreover, the Yamabe minimizer $g \in \gamma$ is unique (up to constant rescaling) whenever $Y_{\gamma} \leq 0$. The situation is much more complicated when $Y_{\gamma}>0$, but it is still not difficult to see that if $g$ is a metric for which $s$ has a fixed sign (positive, zero, or negative) everywhere on $M$, then this sign necessarily agrees with that of the number $Y_{[g]}$.

Yamabe's work was apparently motivated by the hope of constructing Einstein metrics via a variational approach. This idea eventually led Kobayashi [7] and 
Schoen [19] to independently introduce the smooth manifold invariant

$$
\mathcal{Y}(M)=\sup _{\gamma} Y_{\gamma}=\sup _{\gamma} \inf _{g \in \gamma} \frac{\int_{M} s_{g} d \mu_{g}}{\left(\int_{M} d \mu_{g}\right)^{\frac{n-2}{n}}} .
$$

By construction, this is a diffeomorphism invariant of $M$, and is now commonly known as the Yamabe invariant of $M$; note, however, that Schoen called $\mathcal{Y}(M)$ the sigma constant, and that this terminology is still preferred by some authors. Notice that $\mathcal{Y}(M) \leq 0$ iff $M$ does not admit metrics of positive scalar curvature, and that, when this happens, $\mathcal{Y}(M)$ is simply the supremum of the scalar curvatures of unitvolume constant-scalar-curvature metrics on $M$.

The fact that there is some fundamental relation between the Yamabe invariant $\mathcal{Y}(M)$ and Perelman's $\bar{\lambda}$ invariant was probably first pointed out by Anderson [1]. More recently, an e-print by Fang and Zhang [4] computed the Perelman invariant for a large class of 4-manifolds where the Yamabe invariants had already been computed by the present authors $[9,5]$ and others $[14,15]$, and, as was later emphasized by Kotschick [8], their answers exactly agree with those previously discovered in the Yamabe case. The point of this brief note is to observe that this was no mere matter of coincidence:

Theorem A. Suppose that $M$ is a smooth compact $n$-manifold, $n \geq 3$. Then

$$
\bar{\lambda}(M)= \begin{cases}\mathcal{Y}(M) & \text { if } \mathcal{Y}(M) \leq 0 \\ +\infty & \text { if } \mathcal{Y}(M)>0\end{cases}
$$

In fact, this will follow easily once we clearly understand the behavior of $\lambda V^{2 / n}$ on each individual conformal class.

Proposition 1. Suppose that $\gamma$ is a conformal class on $M$ which does not contain a metric of positive scalar curvature. Then

$$
Y_{\gamma}=\sup _{g \in \gamma} \lambda_{g} V_{g}^{2 / n}
$$

Proof. Let $g \in \gamma$, and let $\hat{g}=u^{4 /(n-2)} g$ be the Yamabe minimizer in $\gamma$. Then

$$
0 \geq Y_{\gamma}=\frac{\int_{M}\left[s_{g} u^{2}+4 \frac{n-1}{n-2}|\nabla u|^{2}\right] d \mu_{g}}{\left(\int_{M} u^{2 n /(n-2)} d \mu_{g}\right)^{(n-2) / n}}
$$


Thus

$$
\begin{aligned}
\lambda_{g} \int u^{2} d \mu & \leq \int\left[s u^{2}+4|\nabla u|^{2}\right] d \mu \\
& \leq \int\left[s u^{2}+4 \frac{n-1}{n-2}|\nabla u|^{2}\right] d \mu \\
& =Y_{\gamma}\left(\int u^{2 n /(n-2)} d \mu\right)^{(n-2) / n} \\
& \leq Y_{\gamma} V^{-2 / n} \int u^{2} d \mu
\end{aligned}
$$

where, since $Y_{\gamma} \leq 0$, the last step is an the application of the Hölder inequality

$$
\int f_{1} f_{2} d \mu \leq\left(\int\left|f_{1}\right|^{p} d \mu\right)^{1 / p}\left(\int\left|f_{2}\right|^{q} d \mu\right)^{1 / q}, \frac{1}{p}+\frac{1}{q}=1,
$$

with $f_{1}=1, f_{2}=u^{2}, p=n / 2$, and $q=n /(n-2)$. Moreover, equality holds precisely when $u$ is constant — which is to say, precisely when $g$ has constant scalar curvature.

Since this shows that

$$
\lambda_{g} V^{2 / n} \leq Y_{\gamma}
$$

for every $g \in \gamma$, and since equality occurs if $g$ is the Yamabe minimizer, it follows that

exactly as claimed.

$$
\sup _{g \in \gamma} \lambda_{g} V_{g}^{2 / n}=Y_{\gamma}
$$

We now need make only one more simple observation:

Lemma 2. If $M$ carries a metric with $s>0$, then $\bar{\lambda}(M)=+\infty$.

Proof. Given such a manifold $M$ and any smooth non-constant function $f: M \rightarrow$ $\mathbb{R}$, Kobayashi [7] has shown that there exists a unit-volume metric on $M$ with $s=f$. In particular, given any real number $L$, there is a unit-volume metric $g_{L}$ on $M$ with $s>L$ everywhere. But for such a metric, $\lambda>L$ and $V=1$. Thus, taking $L \rightarrow \infty, \bar{\lambda}(M)=\sup _{g} \lambda_{g} V_{g}^{2 / n}=+\infty$.

Theorem $\mathrm{A}$ is now follows immediately. Indeed, if $\mathcal{Y}(M)>0, M$ admits a metric with $s>0$, and Lemma 2 therefore tells us that $\bar{\lambda}(M)=+\infty$. Otherwise, no conformal class contains a metric of positive scalar curvature, and Proposition 1 therefore tells us that each constant-scalar-curvature metric maximizes $\lambda V^{2 / n}$ in its conformal class. Given an arbitrary maximizing sequence $\hat{g}_{j}$ for $\lambda V^{2 / n}$, we may thus, by conformal rescaling, construct a new maximizing sequence $g_{j}$ consisting of unit-volume constant-scalar-curvature metrics. But for any such sequence, the 
numbers $s_{g_{j}}$ may be viewed either as $\left\{\lambda_{g_{j}} V_{g_{j}}^{2 / n}\right\}$ or as $\left\{Y_{\left[g_{j}\right]}\right\}$. Thus the suprema over the space of all Riemannian metrics of $Y_{[g]}$ and $\lambda_{g} V_{g}^{2 / n}$ must precisely coincide.

Now, there is a substantial literature $[5,9,10,14,15,16]$ concerning manifolds of non-positive Yamabe invariant, and the exact value of the invariant is moreover known for large numbers of such manifolds. By virtue of Theorem A, all of these facts about $\mathcal{Y}(M)$ may therefore immediately be interpreted as instead pertaining to $\bar{\lambda}(M)$.

On the other hand, we have also seen that the Perelman invariant jumps to positive infinity whenever there is a metric of positive scalar curvature. By contrast, the Yamabe invariant always remains finite; indeed, one of Aubin's fundamental contributions to the theory of the Yamabe problem is the fact that $\mathcal{Y}(M) \leq \mathcal{Y}\left(S^{n}\right)$ for any smooth compact $n$-manifold $M$. This systematic discrepancy fundamentally reflects the fact that constant-scalar-curvature metrics are generally not minimizers in the positive case $[7,18]$, and that, moreover, constant-scalar-curvature metrics of arbitrarily high energy exist in profusion [17] in this setting. Thus, while we have found a fascinating link between Perelman's invariant and the Yamabe problem, this observation actually teaches us nothing at all precisely in the regime where the Yamabe problem is the most technically difficult and, in some respects, still the most poorly understood.

\section{References}

[1] M. T. Anderson, Canonical metrics on 3-manifolds and 4-manifolds. Asian J. Math. 10, 127-163 (2006).

[2] T. Aubin, Équations différentielles non linéaires et problème de Yamabe concernant la courbure scalaire. J. Math. Pures Appl. 55(9), 269-296 (1976).

[3] T. Aubin, Some Nonlinear Problems in Riemannian Geometry, Springer Monographs in Mathematics. Springer-Verlag, Berlin, 1998.

[4] F. Fang And Y. Zhang, Perelman's $\lambda$ functional and the Seiberg-Witten equations. e-print math.FA/0608439.

[5] M. Ishida And C. LeBrun, Curvature, connected sums, and Seiberg-Witten theory. Comm. Anal. Geom. 11, 809-836 (2003).

[6] B. Kleiner and J. Lott, Notes on Perelman's papers. e-print math.DG/0605667.

[7] O. Kobayashi, Scalar curvature of a metric of unit volume. Math. Ann. 279, 253-265 (1987).

[8] D. Kotschick, Monopole classes and Perelman's invariant of four-manifolds. e-print math.DG/0608504.

[9] C. LeBrun, Four-manifolds without Einstein metrics. Math. Res. Lett. 3, 133-147 (1996).

[10] C. LeBrun, Ricci curvature, minimal volumes, and Seiberg-Witten theory. Inv. Math. 145 279-316 (2001).

[11] J. Lee And T. PArker, The Yamabe problem. Bull. Am. Math. Soc. 17, 37-91 (1987).

[12] G. Perelman, The entropy formula for the Ricci flow and its geometric applications. e-print math.DG/0211159.

[13] G. Perelman, Ricci flow with surgery on three-manifolds. e-print math.DG/0303109.

[14] J. Petean, Computations of the Yamabe invariant. Math. Res. Lett. 5, $703-709$ (1998). 
[15] J. Petean, The Yamabe invariant of simply connected manifolds. J. Reine Angew. Math., 523, 225-231 (2000)

[16] J. Petean and G. Yun, Surgery and the Yamabe invariant. Geom. Funct. Anal. 9, 1189-1199 (1999).

[17] D. Pollack, Nonuniqueness and high energy solutions for a conformally invariant scalar equation. Comm. Anal. Geom. 1, 347-414 (1993).

[18] R. Schoen, Conformal deformation of a Riemannian metric to constant scalar curvature. J. Differential Geom. 20, 478-495 (1984).

[19] R. Schoen, Variational theory for the total scalar curvature functional for Riemannian metrics and related topics. Lec. Notes Math. 1365, 120-154 (1987).

[20] N. Trudinger, Remarks concerning the conformal deformation of metrics to constant scalar curvature. Ann. Scuola Norm. Sup. Pisa. 22, 265-274 (1968).

[21] H. YAmaBe, On the deformation of Riemannian structures on compact manifolds. Osaka Math. J. 12, 21-37 (1960).

Kazuo Akutagawa, Dept. Mathematics, Tokyo Univ. of Science, Noda 278-8510, Japan e-mail: akutagawa_kazuo@ma.noda.tus.ac.jp

Masashi IshidA, Department of Mathematics, SUNY, Stony Brook, NY 11794-3651, USA

Permanent Address: Department of Mathematics, Sophia University, Tokyo 102-8554, Japan

e-mail: ishida@mm.sophia.ac.jp

Claude LeBrun*, Department of Mathematics, SUNY, Stony Brook, NY 11794-3651, USA

e-mail: claude@math.sunysb.edu

Received: 6 October 2006

Revised: 17 October 2006 